\title{
3. EXECUTIVE SUMMARY
}

\section{Introduction}

In 2004, the NSW Department of Health, in conjunction with the 8 area health services, completed the third year of the New South Wales Population Health Survey, an ongoing survey of the health of people of New South Wales using computer assisted telephone interviewing (CATI). The main aims of the survey are to provide detailed information on the health of the people of New South Wales, and to support the planning, implementation, and evaluation of health services and programs in New South Wales. This 2004 Report on Adult Health from the New South Wales Population Health Survey reports on the health of residents aged 16 years and over.

The content of New South Wales Population Health Survey in 2004 was developed by the Health Survey Program in consultation with key stakeholders, area health services, other government departments, and a range of experts. The content covered the 8 priority areas outlined in Healthy People 2005: New Directions for Public Health in New South Wales. The questionnaire was translated into 5 languages: Arabic, Chinese, Greek, Italian, and Vietnamese.

In 2004, interviews were carried out continuously between February and December. The target population for the adult report was all New South Wales residents aged 16 years and over living in households with private telephones. Households were sampled using list assisted random digit dialling. When a household was contacted, one person was randomly selected for interview. Information for the adult report was collected on approximately 9,800 adults.

\section{Health behaviours}

Unhealthy behaviours contribute significantly to the burden of death and ill health in New South Wales. Health behaviours measured in adults in the New South Wales Population Health Survey in 2004 included alcohol intake, bowel cancer screening, fruit and vegetable consumption, immunisation, physical activity, sexual health, smoking, smoking in the home, and sun protection.

More than one-third of the overall adult population reported undertaking alcohol risk-drinking behaviours. More adult males than adult females reported risk-drinking behaviours, and young adults of both sexes were more likely to report risk-drinking behaviour than the general adult population. There was geographic variation, with rural residents reporting higher levels of risk-drinking than urban residents. Encouragingly, there has been a decrease in the proportion of adults reporting risk-drinking behaviours since 1997.

Among adults aged 50 years and over, 26 per cent reported having a screening test for bowel cancer (either a faecal occult blood test, or a sigmoidoscopy, or a colonoscopy) within the last 5 years. The proportion of women aged between 50 and 69 years being screened for breast cancer in the last 2 years has remained unchanged (74.4 per cent). However, the proportion of women having a Pap test within the last 2 years as a screen for cervical cancer has decreased from 77.3 per cent in 1998 to 72.8 per cent in 2004.

Over three-quarters of people aged 65 years and over reported being vaccinated against influenza in the past 12 months. Less than one-half of people in this age group reported being vaccinated against pneumococcal disease in the preceding 5 years. Up until 2003, the proportion of people being vaccinated against both these conditions continued to increase each year. However, in 2004, the proportion being vaccinated had plateaued.

Just under one-half of all respondents reported eating the recommended daily fruit intake ( 2 serves), while only one in 10 respondents reported consuming the recommended daily minimum quantity of vegetables ( 5 serves). Under one-half of the respondents reported using low fat milk. A greater proportion of females than males consumed the recommended amount of fruit and vegetables and used low fat milk each day. Overall, just under 6 per cent of respondents reported that they had run out of food and could not afford to buy more on at least one occasion in the previous 12 months.

Just over one-half of all respondents aged 16 years and over reported undertaking adequate levels of physical activity (a total of 150 minutes per week on 5 separate occasions). The proportion of males undertaking adequate physical activity was greater than females.

Information on sexual health was collected for the first time in 2004. Around one in 25 people aged 16-70 years reported that they had practised unsafe sex in the previous 12 months. In the 16-24 year age group one in 10 people practised unsafe sex.

In 2004, one in 5 people were still current smokers. For the first time since 1997 there was no difference in the proportion of current smokers between males and females. More than 80 per cent of respondents reported that their home was smoke-free, while 7.5 per cent reported people 'occasionally' smoked inside the house, and 8 per cent reported that people 'frequently' smoked inside the house.

In 2004, an index of sun protection was developed to describe sun protection practices. Just over two-thirds of the adult population scored 'high' on the sun protection index. People were also asked about the ease of finding shade, and almost two-thirds found shade easily at local sporting areas, and around three-quarters found shade easily in parks and swimming pools.

\section{Health status}

In 2004, the New South Wales Population Health Survey collected information from adults on a range of health indicators including self-rated health status, asthma, diabetes, 
hearing, interpersonal injury, oral health, overweight and obesity, psychological distress, and vision.

Almost 80 per cent of the adult population rated their own health as 'excellent', 'very good', or 'good'. This figure has continued to decline since 1997.

Overall, 10 per cent of the adult population aged 16 years and over reported current doctor-diagnosed asthma. A greater proportion of females than males reported current asthma.

Approximately 6 per cent of adults aged 16 years and over reported that a doctor had ever told them that they had diabetes. More males than females reported diabetes. The prevalence of diabetes increased with age and has increased since 1997.

Just over 6 per cent of all respondents reported that they had none of their natural teeth. A greater proportion of females than males had none of their natural teeth.

Just under one-half of all respondents reported being either overweight or obese, and 15 per cent of adults were classified as obese. A significantly greater proportion of males than females were classified as overweight or obese. The proportion of adults classified as overweight or obese has risen since 1997.

Overall, one in 9 respondents reported either 'high' or 'very high' levels of psychological distress. Females were more likely than males to report 'high' or 'very high' levels of psychological distress. Rates of 'high' and 'very high' psychological distress rose significantly between 2003 and 2004.

For the first time, information on hearing and vision was collected. Almost three-quarters of the adult population had had their eyesight tested in the previous 2 years, and just over one-half reported that they had normal vision in both eyes. More males than females had normal vision. Over 80 per cent of the adult population have normal hearing in both ears. Only one in 7 adults with abnormal hearing were using a hearing aid.

Information on interpersonal violence in young people was also collected in 2004. One in 8 adults aged between 16 and 24 years had been the victim of a violent attack in the past 12 months. More males than females reported being attacked.

\section{Health services}

In 2004, the New South Wales Population Health Survey collected information on the use of, and satisfaction with, health services including emergency departments, hospitals, community health centres, and public dental services; and information on difficulties obtaining health care when needed.

Over one in 8 adults reported experiencing difficulties getting health care when needed. Rural residents were more likely to have difficulties getting health care than urban residents. The most frequently reported difficulty was waiting time for an appointment with a general practitioner followed by waiting time for dental services.

One in 7 adults reported attending an emergency department in the previous 12 months; of these, almost four-fifths rated the care received as 'excellent', 'very good', or 'good'. Similarly, one in 7 adults had been admitted to hospital and over 90 per cent of these rated the care received as 'excellent', 'very good', or 'good'. Around 7 per cent of the adult population attended a community health centre, with over 91 per cent rating the care they received as 'excellent', 'very good', or 'good'. Just over 5 per cent attended a public dental service, and 84 per cent rated the care they received as 'excellent', 'very good', or 'good'. 